\title{
Quantum Metrology with Two-Mode Squeezed Vacuum: Parity Detection Beats the Heisenberg Limit
}

\author{
Petr M. Anisimov * Gretchen M. Raterman, Aravind Chiruvelli, William \\ N. Plick, Sean D. Huver, Hwang Lee, and Jonathan P. Dowling \\ Hearne Institute for Theoretical Physics and Department of Physics and Astronomy \\ Louisiana State University, Baton Rouge, LA 70803
}

(Dated: October 31, 2018)

\begin{abstract}
We study the sensitivity and resolution of phase measurement in a Mach-Zehnder interferometer with two-mode squeezed vacuum ( $\bar{n}$ photons on average). We show that super-resolution and subHeisenberg sensitivity is obtained with parity detection. In particular, in our setup, dependence of the signal on the phase evolves $\bar{n}$ times faster than in traditional schemes, and uncertainty in the phase estimation is better than $1 / \bar{n}$.
\end{abstract}

PACS numbers: 07.60.Ly, 95.75.kK, 42.50.-p, 42.50.St

Different physical mechanisms contribute to phase measurement. Thus, measuring phase provides insight into a number of physical processes. Therefore, improved phase estimation benefits multiple areas of scientific research, such as quantum metrology, imaging, sensing, and information processing. Consequently, enormous efforts have been devoted to improve the resolution and sensitivity of interferometers. Sensitivity is a measure of the uncertainty in the phase estimation, while resolution is rate at which signal changes with changing phase.

In what follows, we direct our attention to quantum interferometry. The benchmark that quantum interferometry is compared against is one with coherent light input and intensity difference measurement at the output of a Mach-Zehnder interferometer (MZI). In general, phase sensitivity of this benchmark is shot-noise limited, namely $\Delta \varphi=1 / \sqrt{\bar{n}}$, where $\bar{n}$ is the average number of photons. However, better sensitivity is possible if nonlinear interaction between photons in the MZI takes place [1]. In what follows, we only consider phase accumulation due to linear processes.

In 1981, Caves pointed out that by using coherent light and squeezed vacuum one could beat the shot-noise limit $\Delta \varphi<1 / \sqrt{\bar{n}}$ (super-sensitivity) [2]. In the work of Boto et al., it was shown that by exploiting quantum states of light, such as N00N states, it is possible to beat the Rayleigh diffraction limit in imaging and lithography (super resolution) while also beating the shot-noise limit in phase estimation [3, 4, 5, 6]. Finally, it was shown in Ref. 7] that input state entanglement is important in order to achieve super-sensitivity in a linear interferometer.

non-classical light Experimental realization of these predictions have been hindered by the fact that entangled states of light, with large numbers of photons, are difficult to obtain. Therefore we turn our attention to the brightest (experimentally available) nonclassical light - two-mode squeezed vacuum (TMSV). A state of TMSV is a superposition of twin Fock states $\left|\psi_{\bar{n}}\right\rangle=\sum_{n=0}^{\infty} \sqrt{p_{n}(\bar{n})}|n, n\rangle$, where the probability of a twin Fock state $|n, n\rangle=|n\rangle_{A}|n\rangle_{B}$ to be present de-

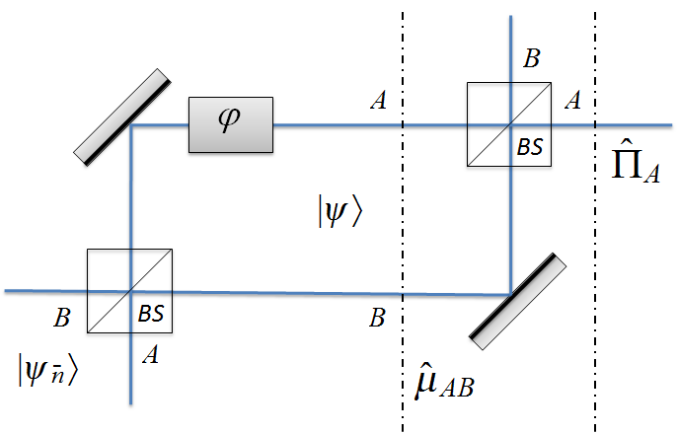

FIG. 1: Mach-Zehnder interferometer used in the calculations. Two-mode squeezed vacuum input state $\left|\psi_{\bar{n}}\right\rangle$ is indicated together with the intermediate state $|\psi\rangle=\hat{P}_{\varphi} \hat{U}\left|\psi_{\bar{n}}\right\rangle$. Vertical dash-dot lines indicate places where two measurements $\hat{\mu}_{A B}$ and $\hat{\Pi}_{A}$ are to be implemented.

pends on average number of photons in both modes of TMSV, $\bar{n}$, in the following way $p_{n}(\bar{n})=\left(1-t_{\bar{n}}\right) t_{\bar{n}}^{n}$ with $t_{\bar{n}}=1 /(1+2 / \bar{n})[8]$.

Light entering a MZI in TMSV state exits a lossless interferometer in the state $\left|\psi_{\mathrm{f}}\right\rangle=\hat{U}_{\mathrm{MZI}}\left|\psi_{\bar{n}}\right\rangle$, where the MZI is described by the unitary transformation $\hat{U}_{\text {MZI }}$ (Fig. 11). This transformation, in terms of the field operators for the optical modes $\hat{a}$ and $\hat{b}$, is $\hat{U}_{\text {MZI }}=\hat{U} \hat{P}_{\varphi} \hat{U}=\exp \left(\varphi\left(\hat{a}^{\dagger} \hat{b}-\hat{b}^{\dagger} \hat{a}\right) / 2\right)$, where $\hat{P}_{\varphi}=\exp (-i \varphi \hat{G})$ describes accumulation of a phase difference $\varphi$; and $\hat{U}=\exp \left(i \frac{\pi}{4}\left(\hat{a}^{\dagger} \hat{b}+\hat{a} \hat{b}^{\dagger}\right)\right)$ describes the 50 50 beam splitter, with a $\pi / 2$ phase shift for the reflected mode. In a linear medium the generator of phase evolution is $\hat{G}=\left(\hat{n}_{A}-\hat{n}_{B}\right) / 2$, where $\hat{n}_{A}=\hat{a}^{\dagger} \hat{a}$ and $\hat{n}_{B}=\hat{b}^{\dagger} \hat{b}$ are the photon number operators in each mode.

Phase estimation is based on the detection of light at the outputs of MZI. Not all detection schemes are capable of exploiting the full potential of non-classical light to be super-sensitive and super-resolving. For example, intensity difference measurement, which is standard for optical 
interferometry with coherent light, is not phase sensitive at all if TMSV input is used [9]. In our work, we consider parity detection for our measuring scheme. The parity operator on output mode $A$ is $\hat{\Pi}_{A}=\exp \left(i \pi \hat{n}_{A}\right)$. Parity was originally discussed in the context of trapped ions by Bollinger et al. [10] and later adopted for optical interferometry by Gerry and Campos [11, 12]. Super-sensitivity with this detection strategy has been shown for several classes of input states [13]. Finally, parity detection was also shown to allow better than classical resolution with coherent light at the shot-noise limit (SNL) [14].

Parity measurement on mode $A$ at the output of MZI is computed by $\left\langle\hat{\Pi}_{A}\right\rangle=\left\langle\psi_{\mathrm{f}}\left|\hat{\Pi}_{A}\right| \psi_{\mathrm{f}}\right\rangle$. It has been shown in Ref. [14] that the parity measurement on mode $A$ after the final beam splitter is equivalent to the measurement that is constructed from all the $\left|M, M^{\prime}\right\rangle \rightarrow\left|M^{\prime}, M\right\rangle$ projectors as follows [14]

$$
\hat{\mu}_{A B}=\sum_{N=0}^{\infty} \sum_{M=0}^{N}|N-M, M\rangle\langle M, N-M|,
$$

acting on the inner modes of MZI, such that $|\psi\rangle=$ $\hat{P}_{\varphi} \hat{U}\left|\psi_{\bar{n}}\right\rangle$. Our use of the $\hat{\mu}_{A B}$ operator here highlights the fact that parity detection combined with $50-50$ beam splitter provides a measurement scheme that includes all of the phase-carrying off-diagonal terms in the two-mode density matrix [14]. Calculation of $\left\langle\hat{\mu}_{A B}\right\rangle$ simplifies significantly once it is noted that such an operator, as well as a beam splitter, does not change the total number of photons in the state. Thus a lossless MZI with the parity detection scheme does not mix twin Fock states with different number of photons giving:

$$
\left\langle\hat{\Pi}_{A}\right\rangle=\left(1-t_{\bar{n}}\right) \sum_{n=0}^{\infty} t_{\bar{n}}^{n}\left\langle\hat{\Pi}_{A}\right\rangle_{n},
$$

where $\left\langle\hat{\Pi}_{A}\right\rangle_{n}=\left\langle n, n\left|\hat{U}_{\mathrm{MZI}}^{\dagger} \hat{\Pi}_{A} \hat{U}_{\mathrm{MZI}}\right| n, n\right\rangle$ is the expectation value of the parity operator for twin Fock state input. In turn, the expression $\left\langle\hat{\Pi}_{A}\right\rangle_{n}=(-1)^{n} P_{n}(\cos (2 \varphi))$, given in terms of Legendre polynomials $P_{n}$, could be found in Ref. [15]. Finally, one can identify our expression in Eq. (2) with the generating function for Legendre polynomials [16] and arrive to the following:

$$
\left\langle\hat{\Pi}_{A}\right\rangle_{\varphi+\pi / 2}=\left\langle\hat{\mu}_{A B}\right\rangle_{\varphi}=\frac{1}{\sqrt{1+\bar{n}(\bar{n}+2) \sin ^{2} \varphi}},
$$

where an additional $\pi / 2$ phase shift was introduced. Eq. (3) is the central result of this paper and, in what follows, it will be used to study the resolution and sensitivity of our proposed scheme.

Let us compare here the signal outcomes of the TMSV scheme with $\bar{n}=10$ to coherent-state-based optical interferometry with $\bar{n}=100$ (see Fig. 2). Intensity

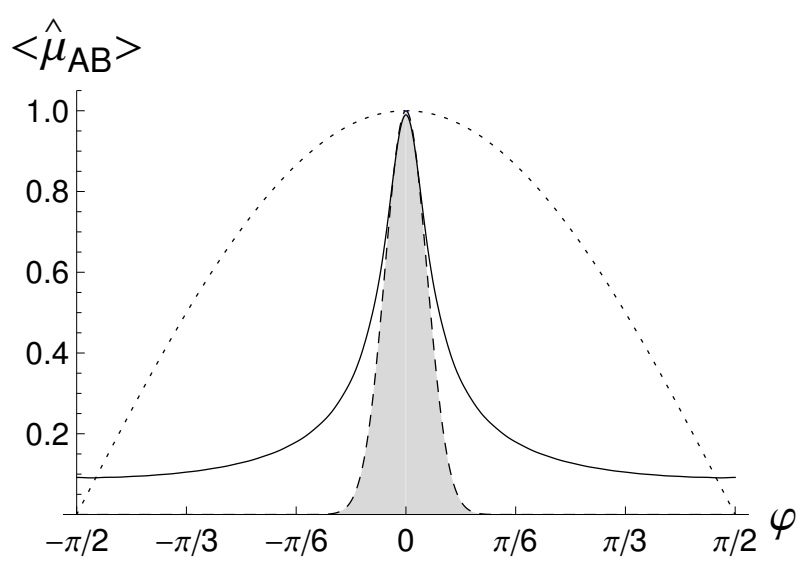

FIG. 2: Measured signals at the output of MZI with coherent light $(\bar{n}=100)$ and TMSV $(\bar{n}=10)$ inputs against accumulated phase difference. Solid and dashed lines are the outputs of parity measurement for TMSV and coherent light, respectively. Dotted line, given for comparison, is a scaled down output of intensity difference measurement on the output of MZI fed with coherent light. TMSV with much smaller photon number performs as well as coherent light.

difference measurement, with coherent state at the input of MZI, exhibits classical interference - a sinusoidal dependence on the phase with an intensity independent period of $2 \pi$. In the case of parity detection with coherent state input, it was shown in Ref. [14] that $\left\langle\hat{\Pi}_{A}\right\rangle=\exp \left(-2 \bar{n} \sin ^{2}(\varphi / 2)\right)$ with a $2 \pi$ period and a feature at the phase origin that is narrower than the classical curve by a factor of $\delta \varphi=1 / \sqrt{\bar{n}}$. In our case, the width of the feature is further reduced by $\sqrt{\bar{n}+2}$ times. Therefore, the peak in Fig. 2 is as narrow for a $\bar{n}=10 \mathrm{TMSV}$ as for a $\bar{n}=100$ coherent state input.

The other aim of optical interferometry is to minimize uncertainty, $\Delta \varphi$, of the measured phase. The lowest bound on the uncertainty is inversely proportional to quantum Fisher information $F_{Q}$ [17]. In the case of a pure state $F_{Q}=4 \Delta \hat{G}^{2}$, where $\Delta \hat{G}^{2}$ is a variance of the phase evolution generator given above. Thus,

$$
\Delta \varphi_{\min }^{2}=\frac{1}{4 \Delta \hat{G}^{2}},
$$

which depends on the state of the light used but not on the measurement. It is in this spirit that we replaced coherent light with two-mode squeezed vacuum in order to beat the sensitivity obtained in Ref. [14]. Our analysis shows that Eq. (4), in the case of coherent light, limits the attainable sensitivity to $\Delta \varphi_{\min }^{2}=\bar{n}^{-1}$, shot noise, while for TMSV it sets much lower limit $\Delta \varphi_{\min }^{2}=$ $(\bar{n}(\bar{n}+2))^{-1}<\bar{n}^{-2}$. This means that TMSV has a potential for super sensitive phase estimation, which has phase uncertainty better than $1 / \bar{n}$ and that is thus subHeisenberg. However, it remains to be seen whether subHeisenberg sensitivity could be obtained with a particu- 


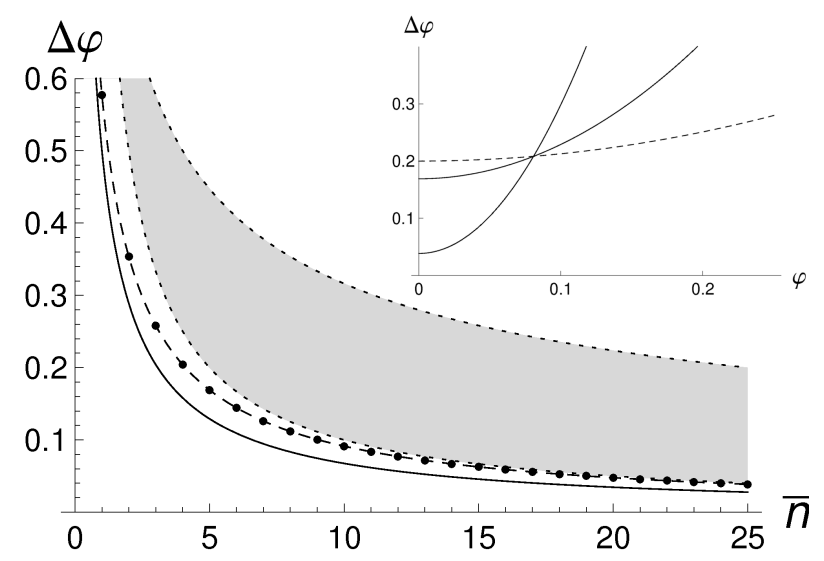

FIG. 3: Sensitivity of phase estimation obtained with parity measurement at $\varphi=0$ (dashed) against average total photon number. Dotes are sensitivity estimation based on quantum Fisher information for integer values of $\bar{n}$. Shaded area is between dotted lines $1 / \bar{n}$ and $1 / \sqrt{\bar{n}}$. Solid line is for the Hofmann limit discussed in the text. Inset: Sensitivity against actual values of accumulated phase difference. Solid lines for TMSV with $\bar{n}=5$ and $\bar{n}=25$; dashed line for coherent light with $\bar{n}=25$.

lar measurement, namely parity.

The variance of the phase estimation based on the outcome of the parity measurement can be estimated as

$$
\Delta \varphi^{2}=\frac{1-\left\langle\hat{\mu}_{A B}\right\rangle^{2}}{\left(\partial\left\langle\hat{\mu}_{A B}\right\rangle / \partial \varphi\right)^{2}},
$$

which is a ratio of detection noise to the rate at which signal changes as function of phase. We have shown that the rate of the signal change is much higher than in the case of coherent state input. Thus, if parity measurement on the squeezed vacuum is no noisier than on the coherent state, sensitivity improvement is expected.

The sensitivity of the phase estimation in our scheme can be estimated based on Eq. (5) combined with Eq. (3):

$$
\Delta \varphi=\frac{1+\bar{n}(\bar{n}+2) \sin ^{2} \varphi}{|\cos \varphi| \sqrt{\bar{n}(\bar{n}+2)}}
$$

which is presented in Fig. 3 for the case of $\varphi=0$. It is clear that in this case sub-Heisenberg sensitivity is obtained and that the lower bound defined by the quantum Fisher information is actually reached. Expression in Eq. (6) gives dependence of the phase sensitivity of our scheme on the actual phase difference as well:

$$
\Delta \varphi \approx \frac{1}{\sqrt{\bar{n}(\bar{n}+2)}}\left(1+(2 \bar{n}(\bar{n}+2)+1) \frac{\varphi^{2}}{2}\right),
$$

where expansion near the phase origin was made. This dependence is, in turn, compared to the one for a coherent state input, which has the following functional dependence on the phase in the vicinity of phase origin

$$
\Delta \varphi \approx \frac{1}{\sqrt{\bar{n}}}\left(1+(2 \bar{n}+1) \frac{\varphi^{2}}{8}\right) .
$$

Dependence of the phase sensitivity in both cases is presented in the inset of Fig. 3 for $\bar{n}=5$ and $\bar{n}=25$ TMSV and for $\bar{n}=25$ coherent state inputs. Comparison shows that our scheme has superior sensitivity in the vicinity of phase origin but degrades rapidly as actual phase difference deviates from zero.

Better than $1 / \bar{n}$ phase sensitivity, demonstrated here by a linear MZI with parity detection and TMSV, is in violation of the so-called Heisenberg limit (HL). In what follows, we will argue why HL is not the true limit, if HL is defined as $\Delta \varphi_{H L}=1 / \bar{n}$.

The traditional argument for the limit on the sensitivity of the phase measurement comes from the Heisenberg uncertainty principle for the phase and photon number $\Delta \varphi \Delta n \geq 1$. This argument is usually combined with intuitive thinking that in the case of finite energy $\Delta n$ should be bounded by $\bar{n}$, that is $\bar{n} \geq \Delta n$. This argument is valid as long as a fixed photon number is assumed $\bar{n}=N$, but has to be treated with caution for fluctuating photon numbers. Such notions about the Heisenberg limit can be traced back to, for example, work by $\mathrm{Ou}$ [18] where he speculates that the fundamental limit set by quantum mechanics on sensitivity is the Heisenberg limit $\Delta \varphi \geq 1 / \bar{n}$ since all analysis up until now had not shown better than $1 / \bar{n}$ sensitivity. Ou's conjecture is in fact not supported by his cited 1986 paper by Yurke et al., on $\mathrm{SU}(1,1)$ interferometers [19], where Eq. (9.31) was published with a typographical error - there is a minus sign where there should be a plus.

Recent progress in quantum metrology has demonstrated the importance of photon number fluctuations for phase estimation. In order to better account for photon number fluctuations, Hofmann in Ref. [20] suggested a more direct definition of the ultimate quantum limit of phase sensitivity $\Delta \varphi^{2} \geq 1 /\left\langle\hat{n}^{2}\right\rangle$, where $\left\langle\hat{n}^{2}\right\rangle$ indicates averaging over the squared photon numbers. Thus, in the case of high photon number fluctuations, $\Delta n^{2}=\left\langle\hat{n}^{2}\right\rangle-\langle\hat{n}\rangle^{2}>0$, the Hofmann limit allows for better sensitivity of the phase measurement than the Heisenberg limit. Clearly $\left\langle\hat{n}^{2}\right\rangle$ contains direct information about fluctuations where $\langle\hat{n}\rangle^{2}$ does not.

In the case of our TMSV with parity, sensitivity of phase estimation is better than allowed by the Heisenberg limit, although marginally, but it is never better than $1 / \sqrt{2 \bar{n}^{2}+2 \bar{n}}$, which is the Hofmann limit. It is also never below the quantum Cramer-Rao lower bound set by the quantum Fisher information of the state at an area of phase accumulation.

In order to demonstrate that the maximal phase sensitivity could be underestimated by the Heisenberg limit if photon number fluctuations are neglected, consider the following state $\hat{\rho}(n, \theta)=\sin ^{2} \theta|0,0\rangle\langle 0,0|+$ $\cos ^{2} \theta|n, n\rangle\langle n, n|$, which has $\bar{n}=2 n \cos ^{2} \theta$. This state could appear in the context of a probabilistic twin Fock state generation with parity detection on a single output mode, since such a detection would not distinguish vac- 


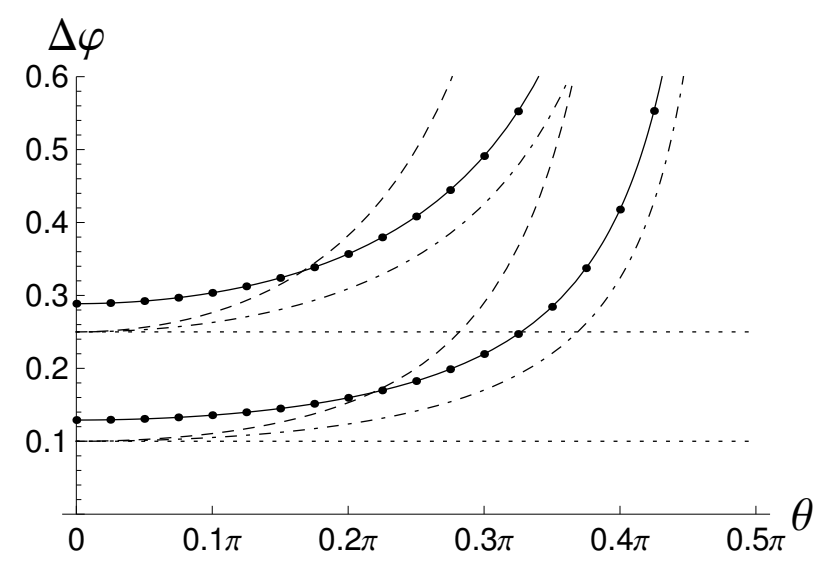

FIG. 4: Phase estimation sensitivity $\Delta \varphi$ for the state $\hat{\rho}(n, \theta)$ with $n=2$ (top four) and $n=5$ (bottom four) against $\theta$. Solid lines represent sensitivity with parity measurement which saturates limit set by quantum Fisher information (dots for a few selected values of $\theta$ ). Dashed lines represent HL sensitivity estimation based on averaged total photon number: $1 / \bar{n}$. Dot-dashed lines give Hofmann's estimation of sensitivity discussed in the text.

uum contribution from the twin Fock contribution when all photons were routed out in the other port.

Based on quantum Fisher information, this state is capable of providing sensitivity $\Delta \varphi^{2}=$ $1 /\left(2 n(n+1) \cos ^{2} \theta\right)$, which is obtainable by parity measurement. This dependence of the phase sensitivity is presented in Fig. 4 for $n=2$ and $n=5$, where the presence of the vacuum, $\theta>0$, degrades the sensitivity but allows for $1 / \bar{n}$ Heisenberg limit to be beat! However, the Hofmann limit $\Delta \varphi^{2}=1 /\left(4 n^{2} \cos ^{2} \theta\right)$ tracks the phase sensitivity well; without being beaten!

There does exist another limit based on the highest number of photons in the state $-1 / N$, with $N=2 n$ for the state considered here. However, it is not as useful as the Hofmann limit for a number of reasons: a) it overestimates the sensitivity as it does for $\hat{\rho}(n, \theta)$; b) information about $N$ is not readily available in experiments; c) for states, such as coherent and squeezed vacuum, $N=\infty$.

Finally, implementation of parity detection needs to be discussed. In proof of principle experiments, a highly efficient photon number-resolving detector could be used. Such detectors with $95 \%$ efficiency and number resolving capabilities in the tens of photons have been demonstrated [21, 22, 23, 24]. However, for more practical applications, knowledge about exact photon numbers is excessive. We conjecture that a scheme, which does not require photon counting, exists, perhaps through the exploitation of optical non-linearities [25], or projective measurements, and this is an area of ongoing research.

In conclusion, the main result of this paper is our demonstration that optical interferometry with twomode squeezed vacuum and parity detection provides an interferometric metrology strategy with sensitivity $\Delta \varphi<\bar{n}^{-1}$ and resolution $\bar{n}^{-1}$ times better than the resolution of standard (classical) interference.

We would like to acknowledge support from the Army Research Office, the Boeing Corporation, the Department of Energy, the Foundational Questions Institute, the Intelligence Advanced Research Projects Activity, and the Northrop-Grumman Corporation.

* Electronic address: []petr@lsu.edu

[1] S. Boixo et al., Phys. Rev. Lett. 101, 040403 (2008).

[2] C. M. Caves, Phys. Rev. D 23, 1693 (1981).

[3] A. N. Boto et al., Phys. Rev. Lett. 85, 2733 (2000).

[4] P. Kok et al., Phys. Rev. A 63, 063407 (2001).

[5] H. Lee, P. Kok, C. Williams, and J. Dowling, J. Opt. B 6 (2004).

[6] J. P. Dowling, Contemp. Phys. 49, 125 (2008).

[7] V. Giovannetti, S. Lloyd, and L. Maccone, Phys. Rev. Lett. 96, 010401 (2006).

[8] C. C. Gerry and P. L. Knight, Introductory Quantum Optics (Cambridge University Press, 2005).

[9] T. Kim, O. Pfister, M. Holland, J. Noh, and J. Hall, Phys. Rev. A 57, 4004 (1998).

[10] J. J. Bollinger, W. M. Itano, D. J. Wineland, and D. J. Heinzen, Phys. Rev. A 54, R4649 (1996).

[11] C. C. Gerry, Phys. Rev. A 61, 043811 (2000).

[12] C. C. Gerry and R. A. Campos, Phys. Rev. A 64, 063814 (2001).

[13] A. Chiruvelli and H. Lee, (2009), arXiv:0901.4395 1 [quant-ph].

[14] Y. Gao, C. F. Wildfeuer, P. M. Anisimov, H. Lee, and J. P. Dowling, (2009), arXiv:0907.2382 v2 [quant-ph].

[15] R. A. Campos, C. C. Gerry, and A. Benmoussa, Phys. Rev. A 68, 023810 (2003).

[16] Handbook of Mathematical Functions, Natl. Bur. Stand. Appl. Math. Ser. No. 55, edited by M. Abramowitz and I. A. Stegun (U.S. GPO, Washington, D.C., 1964), p. 783.

[17] S. L. Braunstein and C. M. Caves, Phys. Rev. Lett. 72, 3439 (1994).

[18] Z. Y. Ou, Phys. Rev. A 55, 2598 (1997).

[19] B. Yurke, S. L. McCall, and J. R. Klauder, Phys. Rev. A 33, 4033 (1986).

[20] H. F. Hofmann, Phys. Rev. A 79, 033822 (2009).

[21] A. J. Miller, S. W. Nam, J. M. Martinis, and A. V. Sergienko, Appl. Phys. Lett. 83, 791 (2003).

[22] A. E. Lita, A. J. Miller, and S. W. Nam, Opt. Express 16, 3032 (2008).

[23] G. Khoury, H. S. Eisenberg, E. J. S. Fonseca, and D. Bouwmeester, Phys. Rev. Lett. 96, 203601 (2006).

[24] C. F. Wildfeuer et al., Phys. Rev. A 80, 043822 (2009).

[25] C. C. Gerry, A. Benmoussa, and R. A. Campos, Phys. Rev. A 72, 053818 (2005). 